\title{
An Improved Natural Field Orientation Control of a Current Fed Induction Machine
}

\author{
G. Mirzaeva and R.E. Betz \\ School of Electrical Engineering and Computer Science \\ University of Newcastle, Callaghan, NSW, Australia, 2308
}

\begin{abstract}
Natural Field Orientation (NFO) is a simplified version of stator flux oriented (SFO) control for an induction machine. The essential difference from traditional SFO is that NFO does not estimate the stator flux magnitude but assumes that it's equal to the reference value. The NFO algorithm only requires knowledge of the stator resistance and is extremely simple to implement. However, it has been found that NFO has stability problems under regeneration. Also when implemented on a current fed induction machine, the control frame has an undesirable tendency to misalign with respect to the stator flux vector. This paper examines both the above issues in detail. It proposes a solution that mitigates this undesirable behaviour and, at the same time, retains the inherent simplicity of the NFO control scheme. The findings of the paper are substantiated by simulation and experimental results.
\end{abstract}

\section{NOMENCLATURF}

$R_{s}, R_{r}$ - stator and rotor resistances;

$L_{m}, L_{s}, L_{r}$ - magnetising, stator and rotor inductances;

$\sigma$ - leakage coefficient, $\sigma=1-L_{m}^{2} /\left(L_{s} L_{r}\right)$;

$T_{r}$ - rotor time constant, $T_{r}=L_{r} / R_{r}$;

$\underline{u}_{s}, \underline{i}_{s}, \underline{e}_{m s}$ - stator voltage, stator current and stator flux voltage space vectors;

$(x, y)$ - axes of the frame aligned with the actual position of the stator flux vector;

$(d, q)$ - control frame aligned with the estimated position of the stator flux vector;

$(\alpha, \beta)$ - axes of the stationary reference frame;

$u_{s x}, u_{s y}, i_{s x}, i_{s y}$ - stator voltage and stator current vectors projections onto $(x, y)$ axes;

$e_{s d}, e_{s q}$ - stator flux voltage projections onto $(d, q)$ axes;

$i_{s d}^{*}, i_{s q}^{*}$ - reference stator currents in the control frame;

$x$ - reference current ratio, $x=i_{s q}^{*} / i_{s d}^{*}$;

$\tau_{e}^{*}$ - reference torque value;

$\left|\underline{i}_{m s}\right|,\left|\underline{i}_{m s}^{*}\right|$ - actual and reference values of the stator magnetising current magnitude;

$\left|\underline{\psi}_{s}\right|,\left|\underline{\psi}_{s}^{*}\right|$ - actual and reference values of the stator flux magnitude;

$\omega_{m s}, \tilde{\omega}_{m s}$ - actual and estimated angular velocity of the stator flux vector;

$\omega_{r}, \omega_{s l}-$ shaft angular velocity (elec. $\mathrm{rad} / \mathrm{sec}$ ), slip frequency (elec. $\mathrm{rad} / \mathrm{sec})=\omega_{m s}-\omega_{r}$;

$\theta_{m s}, \tilde{\theta}_{m s}$ - actual and estimated angular position of the stator flux vector;

$\theta_{e}$ - angular misalignment between $(d, q)$ and $(x, y)$ frames, $\theta_{e}=\tilde{\theta}_{m s}-\theta_{m s}$;

$i_{s d d}$ - additional decoupling stator current added to $i_{s d}$.
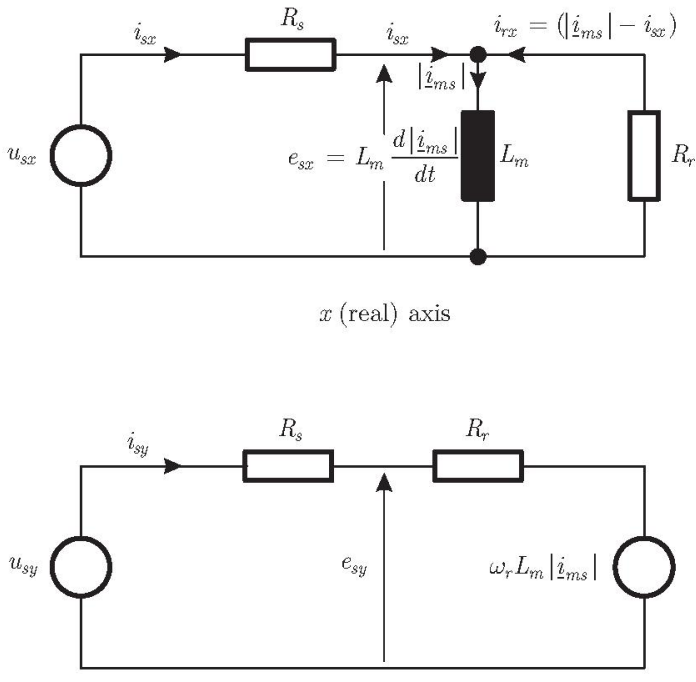

$y$ (imaginary) axis

Fig. 1: Simplified equivalent circuit for the induction machine under stator flux orientation (note: leakage inductances ignored).

\section{INTRODUCTION}

Natural Field Orientation (NFO) is a patented concept that was first developed in the 1980s [1]. The essential idea behind NFO is that if one can sense the stator flux voltage, then assuming that the flux is at a constant value, the stator flux vector will be $90^{\circ}$ spatially lagging this voltage. Therefore one has the position of the flux vector. One does not estimate the stator flux magnitude, as the strategy assumes that the flux is at the reference value. By using the magnitude of the stator flux voltage the angular velocity of the stator flux vector can be estimated. This can then be integrated to give a less noisy estimate of the stator flux position in the machine compared to using the instantaneous value obtained from the stator flux voltage.

The stator flux voltage required for the stator flux estimation can be detected using the following expression:

$$
\underline{e}_{m s}=\underline{u}_{s}-R_{s} \underline{i}_{s} .
$$

This only requires knowledge of one parameter, $R_{s}$, which is less than other schemes that use flux models based on the magnetising and leakage inductances of the machine. Unfortunately reliance on $R_{s}$ also means that the estimate of $\underline{e}_{m s}$ is very sensitive to its accuracy at low speeds.

The NFO algorithm as originally devised [1] controlled a voltage fed machine. An asymmetric version of NFO (voltage 


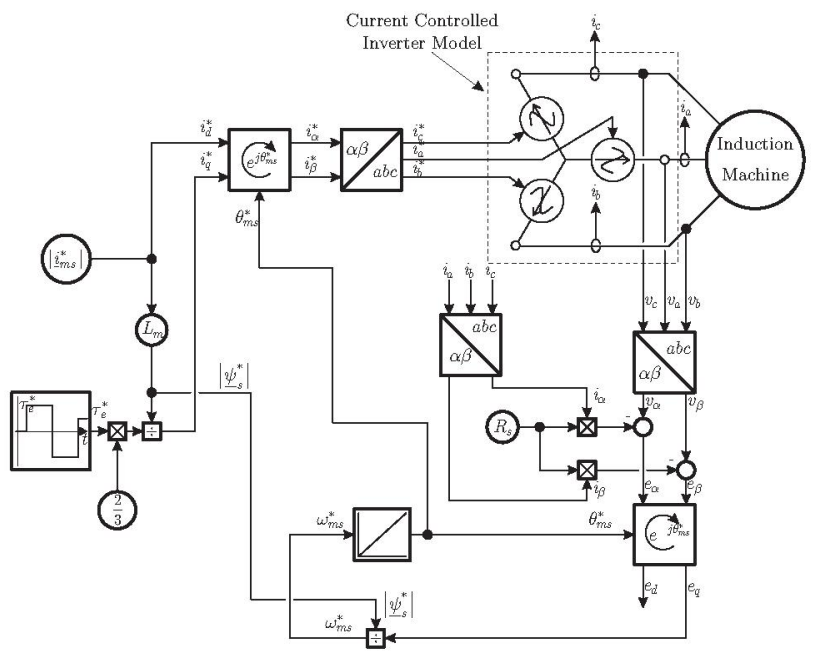

Fig. 2: Block diagram of stator flux oriented NFO algorithm without augmentation.

fed on one axis and current fed on the other) has also been proposed and discussed [2]. However, it can be shown that the NFO algorithm for a current fed machine offers important advantages. It is less sensitive to $R_{s}$ accuracy compared to other techniques and gives a clear indication of an erroneous estimate of the stator flux position (a non-zero value of the d-component of the stator flux voltage). For that reason we shall limit the analysis in this paper to a current fed induction machine under the stator flux NFO control. We shall explore some limitations of this control technique and suggest a solution that offers reasonable performance while retaining the inherent simplicity of the original idea.

The remainder of the paper is organised as follows. Section II outlines the mathematical formulation of the NFO algorithm for an induction machine under the conditions of negligible leakage inductance, and with non-negligible leakage inductance. Issues associated with the static stability of the control frame alignment are investigated for both these cases. Section III proposes and presents simulation results for a solution to the issues identified in Section II. Section IV presents experimental results that validate the proposed solution. Finally Section V presents the conclusions that can be drawn from the results presented in the paper.

\section{NFO FORMULATION FOR A CURRENT FED INDUCTION MACHINE}

\section{A. Machine with negligible leakage}

Neglecting the leakage inductance of the machine results in a very simple NFO formulation. Solving the stator voltage equation in the stator flux oriented frame, where the $x$ axis is aligned with the stator flux vector, results in $[3,4]$ :

$$
\begin{aligned}
& u_{s x}=R_{s} i_{s x}+R_{r}\left(i_{s x}-\left|\underline{i}_{m s}\right|\right) \\
& u_{s y}=R_{s} i_{s y}+R_{r} i_{s y}+\omega_{r} L_{m}\left|\underline{i}_{m s}\right|
\end{aligned}
$$

Similarly, solving the rotor voltage equation under the same conditions gives:

$$
\begin{aligned}
L_{m} \frac{d\left|\underline{i}_{m s}\right|}{d t} & =R_{r}\left(i_{s x}-\left|\underline{i}_{m s}\right|\right) \\
0 & =R_{r} i_{s y}-\left(\omega_{m s}-\omega_{r}\right)\left|\underline{i}_{m s}\right| L_{m}
\end{aligned}
$$

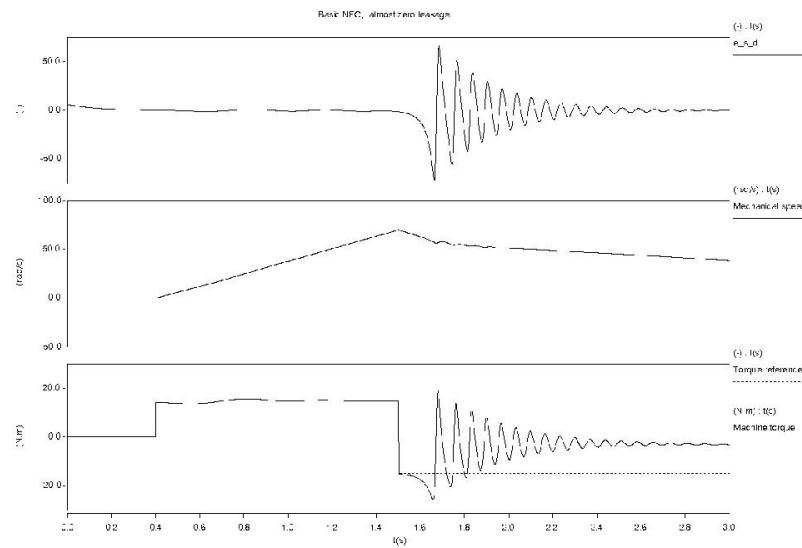

(a) Basic NFO, no leakage

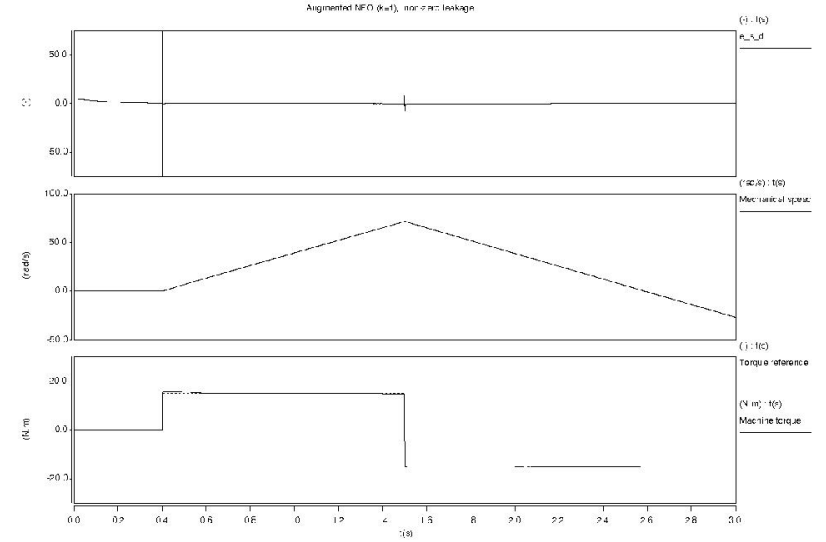

(b) Augmented NFO ( $k=1)$, leakage included

Fig. 3: Simulations of the NFO algorithm.

Combining equations (2) and (3) together one can construct the two axis diagrams for the machine shown in Figure 1. Furthermore if one assumes, as NFO does, that $d\left|\underline{i}_{m s}\right| / d t=0$, then one can write from (2) and (3) the following simplified expressions:

$$
\begin{aligned}
e_{s x} & =0 & i_{s x} & =\left|\underline{i}_{m s}\right| \\
e_{s y} & =\omega_{m s} L_{m}\left|\underline{i}_{m s}\right| & R_{r} i_{s y} & =\omega_{s l} L_{m}\left|\underline{i}_{m s}\right|
\end{aligned}
$$

The key observations that can be made from these expressions are:

- Under the given assumptions, if the control frame is aligned with the stator flux vector, the d-component of the stator flux voltage should be zero.

- The d-component of the stator current reference should be set equal to the reference magnetising current.

- The angular velocity of the stator flux vector is proportional to the quadrature component of the stator flux voltage.

A block diagram of the NFO controller is shown in Figure 2. 
The key expressions in the figure are:

$$
\begin{aligned}
e_{\alpha} & =v_{\alpha}-R_{s} i_{\alpha} & e_{\beta} & =v_{\beta}-R_{s} i_{\beta} \\
e_{s q} & =-e_{\alpha} \sin \tilde{\theta}_{m s}+e_{\beta} \cos \tilde{\theta}_{m s} & & \\
\tilde{\omega}_{m s} & =\frac{e_{s q}}{\left|\underline{\psi}_{s}^{*}\right|}=\frac{e_{s q}}{L_{m}\left|\underline{i}_{m s}^{*}\right|} & \tilde{\theta}_{m s} & =\int \tilde{\omega}_{m s} d t+\theta_{m s}\left(t_{0}\right) \\
i_{s q}^{*} & =\frac{\tau_{e}^{*}}{\left|\underline{\psi}_{s}^{*}\right|}=\frac{\tau_{e}^{*}}{L_{m}\left|\underline{i}_{m s}^{*}\right|} & i_{s d}^{*} & =\left|\underline{\underline{i}}_{m s}^{*}\right|
\end{aligned}
$$

This control algorithm was simulated in Saber ${ }^{\circledR}$ for an induction machine with the following parameters: $L_{m}=0.0828 H$, $L_{l s}=L_{l r}=0.0041 H, R_{s}=0.5 \Omega, R_{r}=0.6 \Omega, P_{p}=2$.

The results of the simulation are presented in Figure $3 \mathrm{a}$ which shows both motoring and regeneration modes of operation. One can see from these figures that the algorithm has good dynamic response and torque reference tracking while motoring, but once it is forced into regeneration mode it completely loses control of the machine. This result is consistent with those obtained by Pulle $[5,6]$.

The following observation assists in analysing the problem of instability in regeneration mode. For the stator flux oriented algorithm to have decoupled and accurate control over the machine, the reference frame in which the computations are performed should be closely aligned with the stator flux vector position. Any difference between the angular positions of the two frames, denoted as $\theta_{e}=\tilde{\theta}_{m s}-\theta_{m s}$, once it occurs, should be implicitly driven back to zero. This requirement in turn means that for $\theta_{e}>0$ its derivative $\frac{d \theta_{e}}{d t}$ should be negative, and for $\theta_{e}<0$, $\frac{d \theta_{e}}{d t}$ should be positive. As $\frac{d \theta_{e}}{d t}=\tilde{\omega}_{m s}-\omega_{m s}$, the following expression can be derived $[3,4]$ :

$$
\begin{aligned}
\frac{d \theta_{e}}{d t}=-\sin \theta_{e}\left(\sin \theta_{e}+\right. & \left.x \cos \theta_{e}\right) \\
& \left(\frac{R_{r}}{L_{m}} \frac{\sin \theta_{e}+x \cos \theta_{e}}{\cos \theta_{e}-x \sin \theta_{e}}+\omega_{r}\right)
\end{aligned}
$$

The crucial parameters in (7) are the ratio of the input current references, defined as $x=i_{s q}^{*} / i_{s d}^{*}$, the frame misalignment $\theta_{e}$ and the shaft angular velocity $\omega_{r}$. Plots for $\frac{d \theta_{e}}{d t}$ as a function of $x$ and $\theta_{e}$, with $\omega_{r}$ as a parameter, were developed for both motoring and regeneration modes. The $k$ value indicated in these figures refers to the feedback gain associated with a augmented version of the algorithm. ${ }^{1}$ Figure $4 a$ indicates stable motoring and Figure $4 \mathrm{~b}$ indicates unstable regeneration for most of the $x$ values (excluding those corresponding to very small torque references).

Remark 1: Stability in the 3D plots can be easily seen when $d \theta_{e} / d t$ is of the opposite sign to $\theta_{e}$. If these two parameters are the same sign then it is statically unstable.

Conclusion 1: NFO in its raw formulation is statically unstable over most of the regeneration region.

\section{B. Machine with non-negligible leakage}

If machine leakage inductance is included in the analysis, cross-coupling between the two axes occurs and should be considered with respect to the SFO/NFO-controlled current

\footnotetext{
${ }^{1}$ See later in this paper for a description
}

fed machine. Solving the stator voltage equation with leakage inclusion gives:

$$
\begin{aligned}
& u_{s x}=R_{s} i_{s x}+\frac{L_{s}}{T_{r}} i_{s x}+\sigma L_{s}\left(\frac{d i_{s x}}{d t}-\omega_{s l} i_{s y}\right)-\frac{L_{m}}{T_{r}}\left|\underline{i}_{m s}\right| \\
& u_{s y}=R_{s} i_{s y}+\frac{L_{s}}{T_{r}} i_{s y}+\sigma L_{s}\left(\frac{d i_{s y}}{d t}+\omega_{s l} i_{s x}\right)+\omega_{r} L_{m}\left|\underline{i}_{m s}\right|
\end{aligned}
$$

Similarly, solving the rotor voltage equation results in:

$$
\begin{aligned}
L_{m} \frac{d\left|\underline{i}_{m s}\right|}{d t}+\frac{L_{m}}{T_{r}}\left|\underline{i}_{m s}\right| & =\sigma L_{s} \frac{d i_{s x}}{d t}+\frac{L_{s}}{T_{r}} i_{s x}-\sigma L_{s} \omega_{s l} i_{s y} \\
\sigma L_{s} \frac{d i_{s y}}{d t}+\frac{L_{s}}{T_{r}} i_{s y} & =\omega_{s l}\left(L_{m}\left|\underline{i}_{m s}\right|-\sigma L_{s} i_{s x}\right)
\end{aligned}
$$

It is clear from the above equations that there exists crosscoupling between $\left|\underline{i}_{m s}\right|$ and $i_{s y}$ via the leakage components. It is shown in [7] that an augmented $d$ axis reference current $i_{d_{r e f}}=i_{s d}^{*}+i_{s d d}$, will decouple this cross-coupling effect. The decoupling current $i_{s d d}$ is given by:

$$
i_{s d d}=\int\left(-\frac{1}{\sigma T_{r}} i_{s d d}+\omega_{s l} i_{s y}\right) d t
$$

where

$$
\omega_{s l}=\frac{L_{s} i_{s y}+\sigma T_{r} L_{s} \frac{d i_{s y}}{d t}}{T_{r}\left(L_{m}\left|\underline{i}_{m s}\right|-\sigma L_{s} i_{s x}\right)} .
$$

Clearly (10) and (11) involve virtually all the parameters of the machine, and using this expression largely defeats many of the desirable attributes of stator flux oriented techniques. One other option to get an approximate value of the slip frequency is to use the $\tilde{\omega}_{m s}$ estimate from the NFO algorithm together with a measurement of the shaft angular velocity. Clearly this would not allow a sensorless algorithm.

For simple implementation and low parameter dependence reasons our first preference would be not to apply the decoupling circuit and see how much negative impact this has on the performance. In a similar manner to the no-leakage inductance case one can solve $\frac{d \theta_{e}}{d t}$ with respect to $x, \theta_{e}$ and $\omega_{r}$ (due to the non-linear nature and complexity of the expression this is done numerically). Plots of $\frac{d \theta_{e}}{d t}$ for motoring and regeneration modes appear in Figure 5a and Figure 5b respectively. One can see that again most of the regeneration region remains unstable. A part of motoring region (where $\theta_{e}<0$ and $\frac{d \theta_{e}}{d t}<0$ ) is now also unstable.

Remark 2: It should be noted that without the decoupling circuit the NFO scheme is unstable for zero frame misalignment. This means that the control frame will be drifting away from the true frame position and will possibly stabilise at some non-zero misalignment angle that depends on torque.

This observation has been confirmed by simulation studies. With respect to Figure 2, the reference d-axis stator current is now assumed to be:

$$
i_{s d}^{*}=\frac{L_{m}}{L_{s}}\left|\underline{i}_{m s}^{*}\right|+i_{s d d}
$$

where $i_{s d d}=0$ if decoupling is not applied and $i_{s d d}$ is estimated based on (10) if decoupling is applied.

It is natural to expect that adding the decoupling circuit would significantly improve the situation. However, one should note that estimation of the slip frequency based on $\tilde{\omega}_{m s}$ or direct 


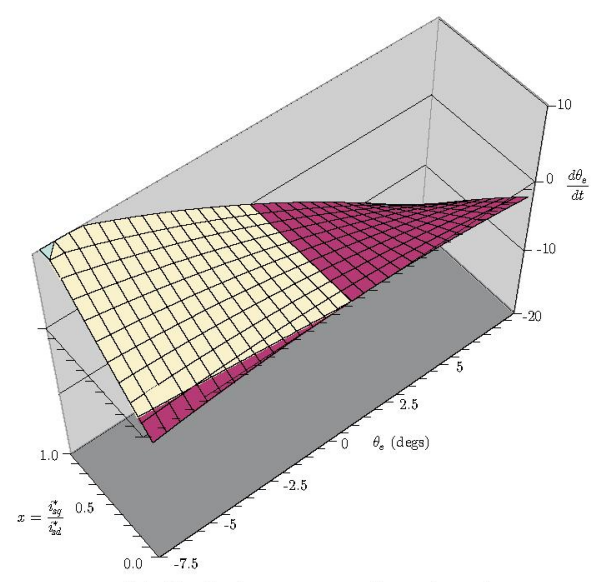

(a) No leakage, motoring, $k=0$

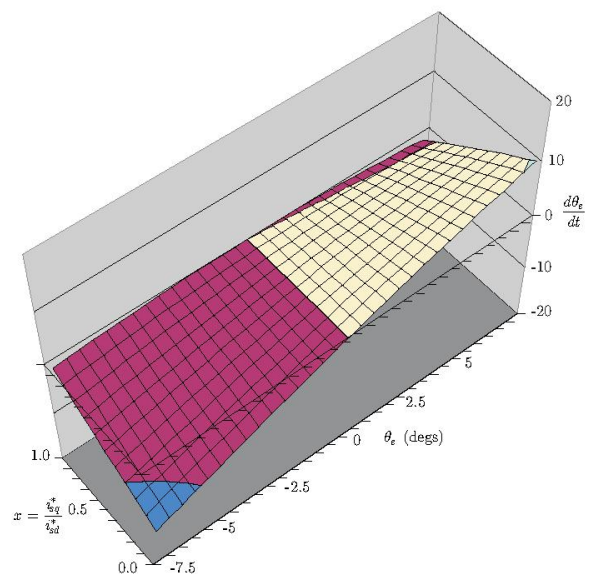

(b) No leakage, regeneration, $k=0$

Fig. 4: NFO frame stability with no leakage and no augmentation $(k=0)$.

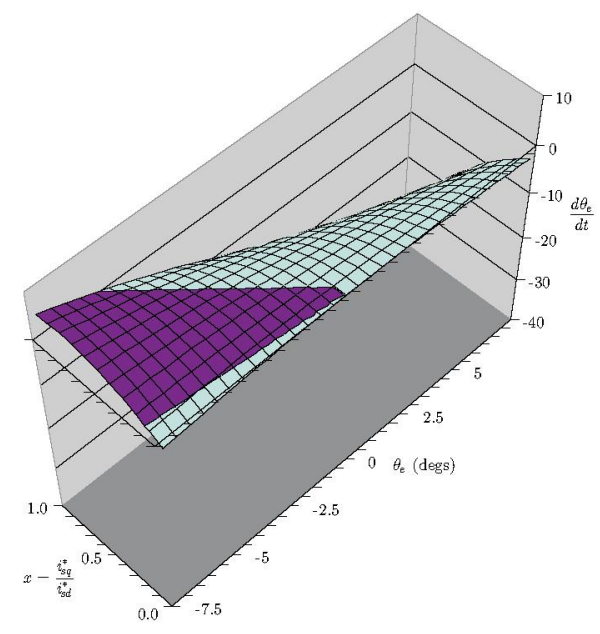

(a) Leakage included, motoring, $k=0$

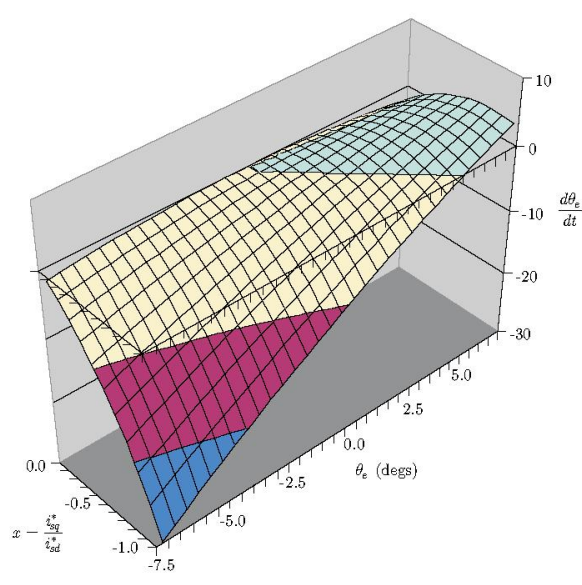

(b) Leakage included, regeneration, $k=0$

Fig. 5: NFO frame stability with leakage and no augmentation $(k=0)$.

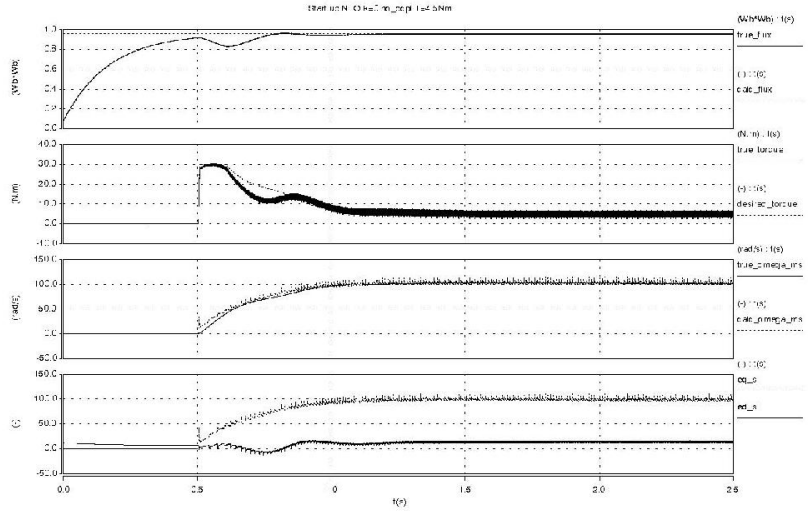

(a) Decoupling circuit is not applied

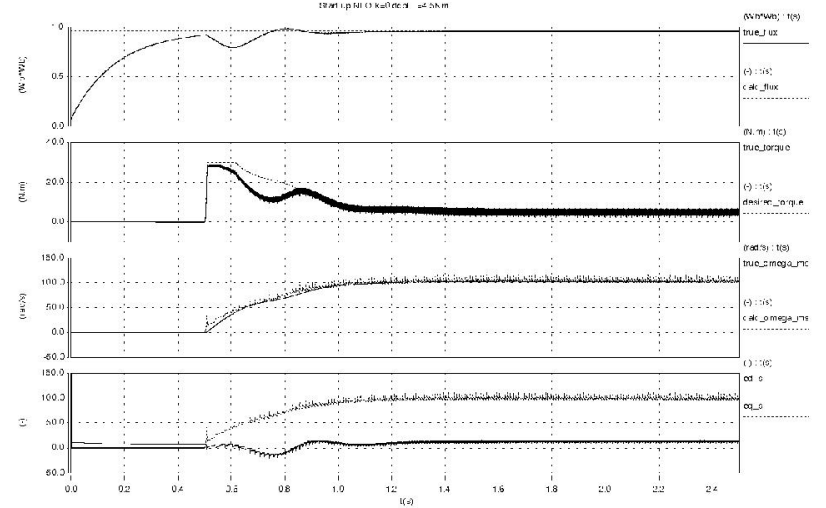

(b) Decoupling circuit is applied

Fig. 6: Simulated start up plots for non-augmented NFO control

$\omega_{s l}$ estimation from the equation (11) are also affected by frame misalignement. Hence the estimated value of the decoupling current $i_{s d d}$ derived in the control frame becomes incorrect with respect to the stator flux vector frame. This compromises potentially positive effect that the decoupling may have on frame alignment.

Figure 6 shows simulation results for start-up of the machine under the NFO control: (a) - with and (b) - without decoupling. One can see minor differences in the dynamics. In both cases the machine reaches steady state with the static frame misalignments of approximately equal magnitudes. This is indicated by non-zero $e_{s d}$ voltage.

Conclusion 2: The addition of the decoupling circuit does not help much with respect to solving the problem of the control 


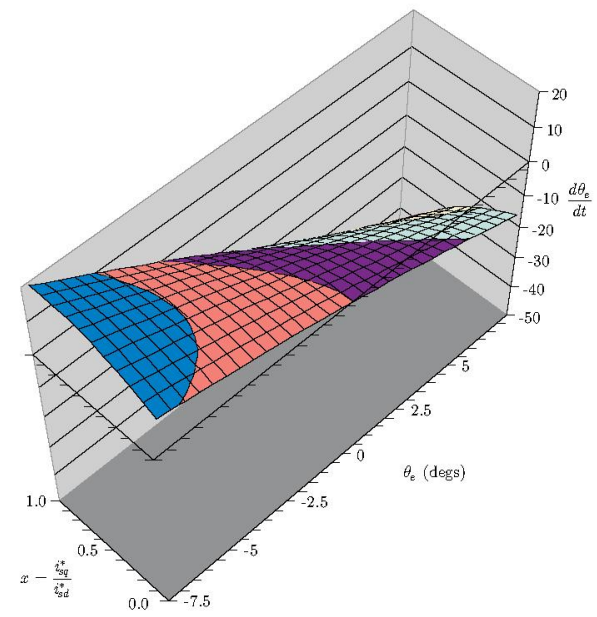

(a) Leakage included, motoring, $k=1$

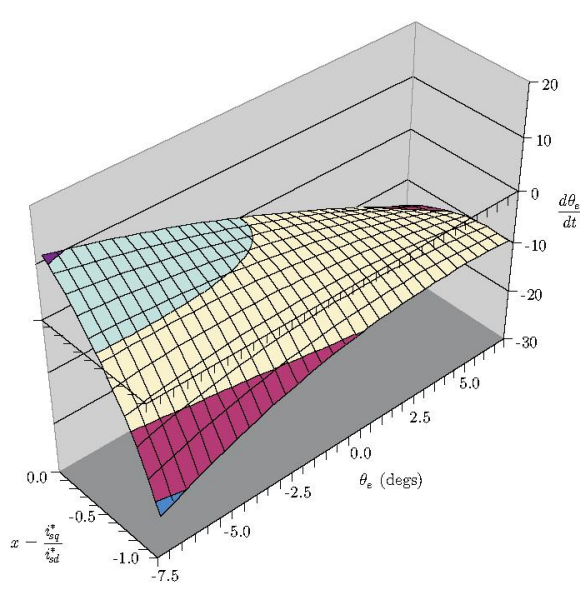

(b) Leakage included, regeneration, $k=1$

Fig. 7: NFO frame stability plots with leakage and augmentation $(k=1)$.

frame drifting away from the stator flux vector frame. Some alternative solution has to be looked for.

Conclusion 3: The addition of leakage inductance in the induction machine model does not substantially change the conclusions drawn from the no leakage case. The frame alignment is still statically unstable over most of the regeneration region, and in addition parts of the motoring region also now exhibit static frame instability.

\section{AUgmented NFO AlgorithM}

The proposed stabilisation strategy for both instability in regeneration mode and the drifting frame in motoring mode is based on perfect alignment being indicated by $e_{s d}=0$. When misalignment of the control frame occurs then a non-zero $e_{s d}$ voltage will appear. The presence of this signal can be used to provide auxiliary feedback that can stabilise the control frame alignment regardless of the source of its disturbance. Using the principle of negative feedback one can develop the following augmentation to the frame angular velocity expression:

$$
\tilde{\omega}_{m s}=\frac{e_{s q}-\operatorname{sign}\left(e_{s q}\right) k e_{s d}}{\left|\underline{\psi}_{s}^{*}\right|}
$$

where $k>0$ is included as a tunable gain parameter.

Plots of $\frac{d \theta_{e}}{d t}$ derived for $k=1$ are shown in Figure $7 \mathrm{a}$ (motoring) and Figure $7 \mathrm{~b}$ (regenerating). It can be seen that a significant part of the regeneration region is now stable, and the drift of the stable position in motoring has been reduced to almost zero. The value of $k$ parameter can be dynamically changed. Furthermore, it can be replaced by a proportionalintegral block in order to improve the control characteristics of the frame alignment feedback.

The block diagram of the augmented NFO with $e_{s d}$ feedback appears in Figure 8. Simulation results for $k=1$ are shown in Figure $3 \mathbf{b}$. A small value of $e_{s d}$ indicates good alignment in both motoring and regenerating modes. The algorithm is stable and provides good tracking and fast response to step changes of the torque reference.

Good dynamic properties and frame alignment are further illustrated by a simulated start-up plot in Figure 9b. The improved performance with augmentation is particularly salient

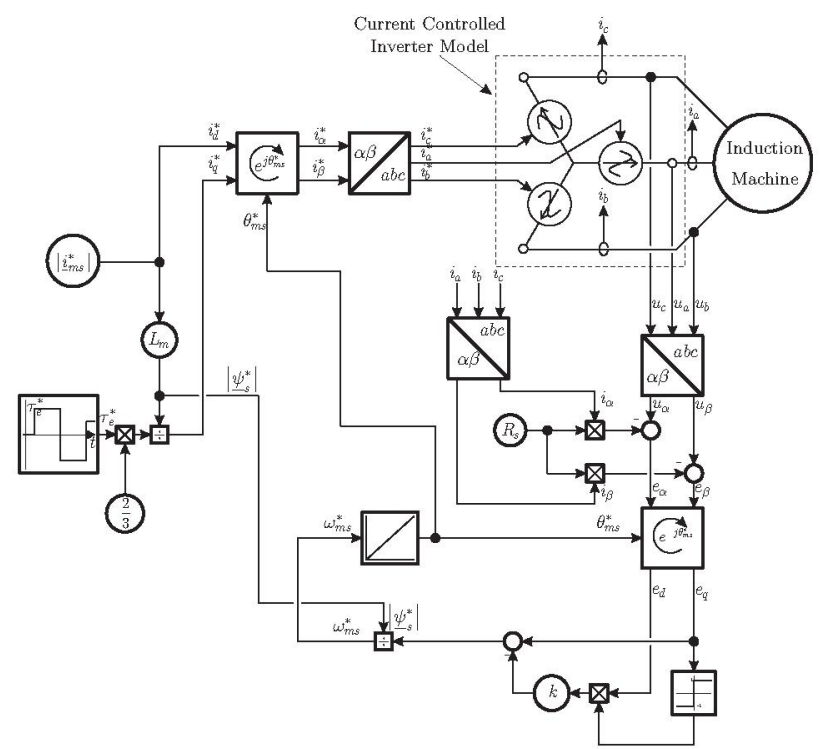

Fig. 8: Block diagram of the stator flux oriented NFO with additional feedback.

when Figure $9 \mathrm{~b}$ is compared to Figure $9 \mathrm{a}$, which is for the nonaugmented NFO.

\section{EXPERIMENTAL RESULTS}

The experiments were carried out using an IGBT based inverter connected to a $7.5 \mathrm{~kW}$ induction machine. This machine was mounted on a dynamometer test bed with a DC load machine configured as a simple Ward-Leonard system to provide static loading capable of regeneration or motoring operation.

The non-augmented version of the NFO algorithm was tested first. In motoring mode it showed a tendency to drift away from the correct frame position, as illustrated by the $e_{s d}$ value in the start-up plots of Figure 9c. After adding the stabilisation feedback (without applying the decoupling circuit) the situation improved significantly. This is illustrated by Figure $9 \mathrm{~d}$ where a much better response to a step change and an almost zero static frame misalignment are readily seen (i.e. $e_{s d} \approx 0$ ).

The steady state operation of non-augmented $(k=0)$ and augmented ( $k=1$ and $k=2$ ) NFO algorithms are compared 


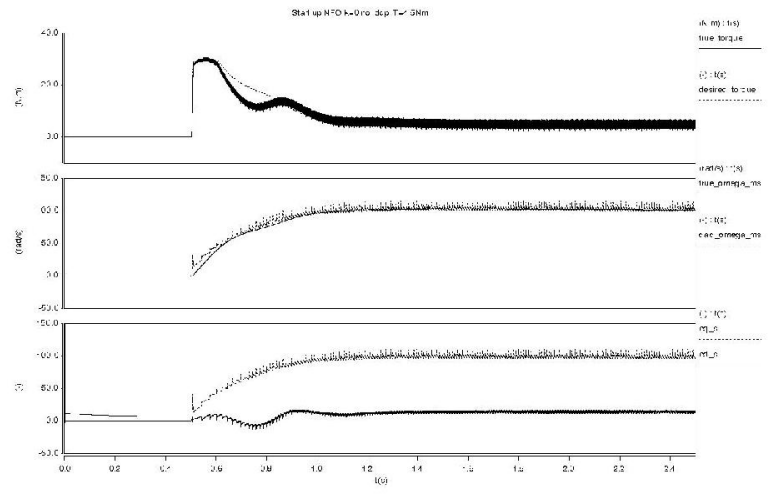

(a) Simulation data, $k=0$
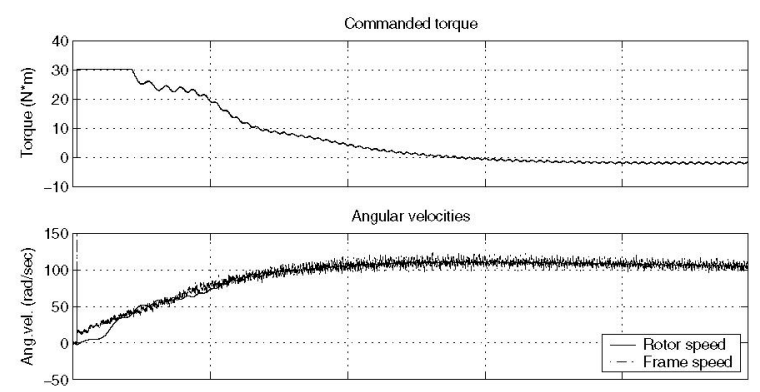

Raw back-emts in SFO frame

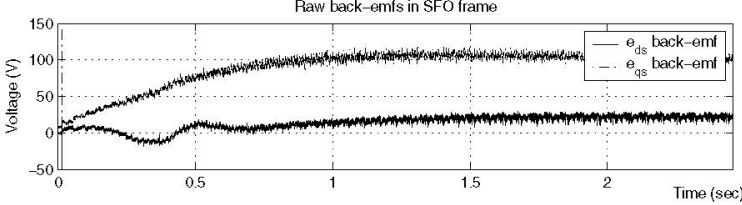

(c) Experimental data, $k=0$

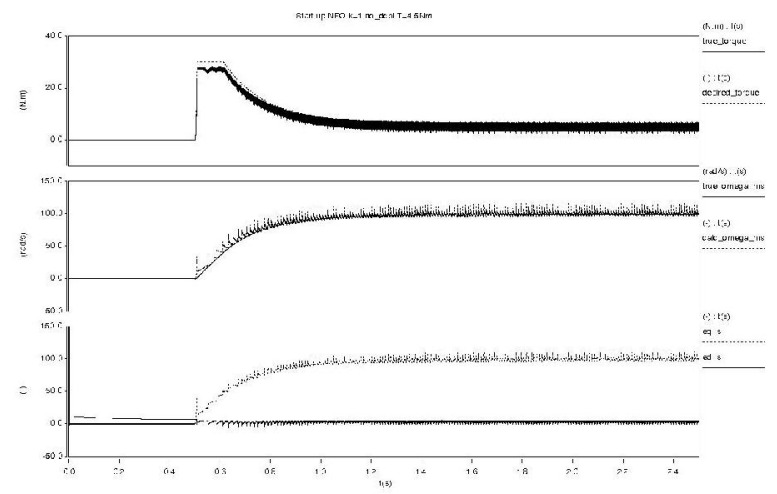

(b) Simulation data, $k=1$
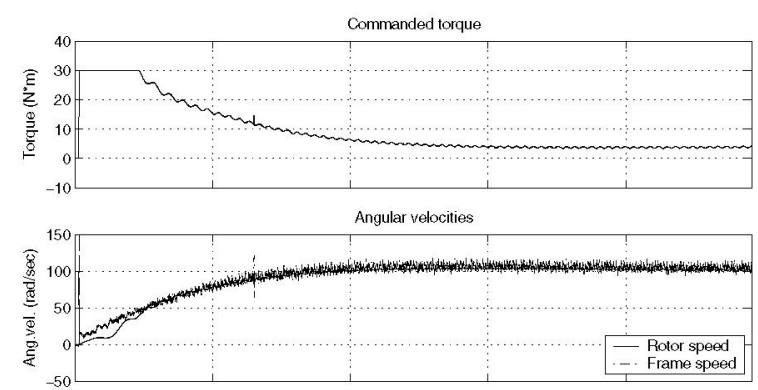

Raw hack-emfs in SFO trame

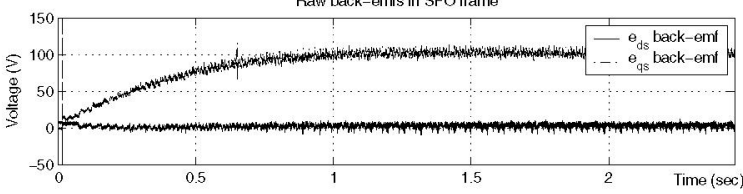

(d) Experimental data, $k=1$

Fig. 9: Simulation and experimental results for non-augmented $(k=0)$ and augmented $(k=1)$ NFO control

\begin{tabular}{|c|c|c|c|c|c|c|}
\hline \multirow{2}{*}{$\begin{array}{c}\text { Torque } \\
\text { applied }\end{array}$} & \multicolumn{2}{|c|}{$\mathbf{k = 0}$} & \multicolumn{2}{c|}{$\mathbf{k = 1}$} & \multicolumn{2}{c|}{$\mathbf{k = 2}$} \\
\cline { 2 - 7 } & $\begin{array}{c}\theta_{e} \\
(\mathrm{deg})\end{array}$ & $\begin{array}{c}\tau_{e}^{*} \\
(\mathrm{Nm})\end{array}$ & $\begin{array}{c}\theta_{e} \\
(\mathrm{deg})\end{array}$ & $\begin{array}{c}\tau_{e}^{*} \\
(\mathrm{Nm})\end{array}$ & $\begin{array}{c}\theta_{e} \\
(\mathrm{deg})\end{array}$ & $\begin{array}{c}\tau_{e}^{*} \\
(\mathrm{Nm})\end{array}$ \\
\hline \hline $4.5 \mathrm{Nm}$ & 13.09 & -1.55 & 1.72 & 4.09 & 1.01 & 4.43 \\
\hline $20.0 \mathrm{Nm}$ & 7.14 & 10.28 & 0.99 & 22.53 & 0.64 & 22.11 \\
\hline$-6.7 \mathrm{Nm}$ & N/A & N/A & -4.93 & -3.44 & -2.65 & -5.42 \\
\hline
\end{tabular}

TABLE I: Summary of the steady state test results

with the help of Table I. A series of three experiments were performed: two for motoring mode and one for regeneration. One can see that the static misalignment reduces and the algorithm's internal estimate of the applied torque becomes more accurate as the parameter $k$ increases.

In addition, the same three feedback gains were tested in regeneration. The non-augmented NFO was unable to work in regeneration, the augmented NFO with $k=1$ was stable in regeneration down to $-12 \mathrm{Nm}$, and with $k=2$ was stable down to $-16 \mathrm{Nm}$. This is just over half the rated torque value of the machine, and corresponds to the observations made from Figure 7.

\section{CONCLUSIONS}

The main conclusions with respect to the frame instability of NFO are:

- The raw NFO algorithm for a machine with a small leakage has a frame angle static instability for most of the regeneration region. This has been demonstrated using theory, simulation and experiments.

- For a machine with a higher leakage, the raw NFO algorithm has a static torque-dependent misalignment between the control frame and the true stator flux vector position. This can be seen in the $3 \mathrm{~d}$ plots of $d \theta_{e} / d t$.

- Regeneration mode can be made statically stable by augmenting the basic NFO algorithm with $\operatorname{sign}\left(e_{s q}\right) \times e_{s d}$ feedback. This has been shown via analysis, simulation and experiments to effectively stabilise the static frame instability present in the basic algorithm.

- The same stabilisation strategy significantly reduces the misalignment angle and dependency of this misalignment on the applied torque.

\section{REFERENCES}

[1] R. Jönsson, "Method and apparatus for controlling an AC motor," United States Patent 4,458,193, July 1984.

[2] D. Pulle, "Analysis of the nfo pm type drive," July 2001, private Report.

[3] R. Betz, G. Mirzaeva, and D. Pulle, "Frame alignment stability issues in natural field orientation," School of Electrical Engineering and Computer Science, University of Newcastle, Australia, Tech. Rep. V1.13, January 2006.

[4] R. Betz and G. Mirzaeva, "Frame alignment stability issues in natural field orientation," in CDROM Proceedings of the IEEE IAS Annual Meeting. IEEE, October 2006.

[5] D. Pulle, "Nfo instability: defining limits, part 2," March 2001, private Report.

[6] _- "Analysis of the nfo type drive," June 2001, private Report.

[7] P. Vas, Vector Control of AC Machines. Oxford University Press, 1990, ISBN:0-19-859370-8. 